\title{
U⿱宀⿻三丨口
}

\section{Learning Behaviour for Service Personalisation and Adaptation}

Chen, L., Skillen, K-L., Burns, W., Quinn, S., Rafferty, J., Nugent, CD., Donnelly, MP., \& Solheim, I. (2014). Learning Behaviour for Service Personalisation and Adaptation. In Learning Behaviour for Service Personalisation and Adaptation. Machine Learning and Cybernetics (pp. 287-297). Springer. https://doi.org/10.1007/978-3-662-45652-1_29

Link to publication record in Ulster University Research Portal

\section{Published in:}

Learning Behaviour for Service Personalisation and Adaptation. Machine Learning and Cybernetics

\section{Publication Status:}

Published (in print/issue): 05/12/2014

DOI:

10.1007/978-3-662-45652-1_29

\section{Document Version}

Author Accepted version

\section{General rights}

Copyright for the publications made accessible via Ulster University's Research Portal is retained by the author(s) and / or other copyright owners and it is a condition of accessing these publications that users recognise and abide by the legal requirements associated with these rights.

\section{Take down policy}

The Research Portal is Ulster University's institutional repository that provides access to Ulster's research outputs. Every effort has been made to ensure that content in the Research Portal does not infringe any person's rights, or applicable UK laws. If you discover content in the Research Portal that you believe breaches copyright or violates any law, please contact pure-support@ulster.ac.uk. 


\title{
LEARNING BEHAVIOUR FOR SERVICE PERSONALISATION \& ADAPTATION
}

\author{
LIMING CHEN ${ }^{1}$, KERRY SKILLEN $^{2}$, WILLIAM BURNS ${ }^{2}$, SUSAN QUINN ${ }^{2}$, \\ JOSEPH RAFFERTY ${ }^{2}$, CHRIS NUGENT ${ }^{2}$, MARK DONNELLY ${ }^{2}$, IVAR SOLHEIM ${ }^{3}$ \\ ${ }^{1}$ School of Computer Science and Informatics, De Montfort University, UK \\ ${ }^{2}$ School of Computing and Mathematics, University of Ulster, UK \\ ${ }^{3}$ Norwegian Computing Center, Norway \\ EMAIL: liming.chen@dmu.ac.uk, \{k.skillen, wp.burns, cd.nugent, mp.donnelly@ulster.ac.uk\}@ulster.ac.uk, \{Quinn-S47, \\ Rafferty-J\}@email.ulster.ac.uk, ivar.solheim@nr.no
}

\begin{abstract}
:
Context-aware applications within pervasive environments are increasingly being developed as services and deployed in the cloud. As such these services are increasingly required to be adaptive to individual users to meet their specific needs or to reflect the changes of their behavior. To address this emerging challenge this paper introduces a service-oriented personalisation framework for service personalisation with special emphasis being placed on behavior learning for user model and service function adaptation. The paper describes the system architecture and the underlying methods and technologies including modelling and reasoning, behavior analysis and a personalisation mechanism. The approach has been implemented in a service-oriented prototype system, and evaluated in a typical scenario of providing personalised travel assistance for the elderly using the help-on-demand services deployed on smartphone.
\end{abstract}

Keywords:

Personalisation; Behavior learning; Adaptation; Pervasive systems; Semantic modelling; Assistive living

\section{Introduction}

Recently, two trends have emerged for context-aware applications in pervasive environments. Firstly, context -aware applications are increasingly being developed as services, which are deployed in the cloud so that they can be shared and reused by large user cohorts in the same application contexts. Secondly, the rapid development of mobile computing technologies in conjunction with the upsurge of smartphone users has driven the application domains from close-world smart environments towards open-world changing environments. In such application scenarios, smartphones provide mobile users with front-end interfaces to interact with embedded sensors and devices, and to deliver functions in changing environments. These two trends have subsequently led to three emerging research questions, namely (i) how the needs of different users are met by the same application, (ii) how the varying needs of one user in different environments are met as they transition from one environment to another, and (iii) how the changing needs of a user are dealt with as and when they become familiar with the application scenarios and develop sufficient knowledge and capabilities.

To address the aforementioned issues context-aware, personalised and just-in-time service provisioning will be key While significant progress has been made in context awareness [7], it still remains a challenge for services to provide the 'right' information for the 'right' user at the 'right' time in the 'right' way. To achieve this, context-awareness alone is insufficient. Personalisation is therefore required to tailor services based on users' unique preferences, needs or capabilities. Comparing to personalisation in web information retrieval [1] and user interface design [2], service personalisation for mobile users in pervasive environments poses a number of challenges. Firstly, a user within different environments typically has varying behaviors. The required services will change dynamically as a user moves around. For example, in a home environment people will usually carry out typical activities related to daily living, while those who travel will have different activities relating to their location or information seeking. Secondly, in a specific environment there may be multiple services each providing a specific service or targeted towards a specific cohort of users. For example, in a smart care home, services could be provided for dementia patients, diabetes patients or the elderly, and each patient may have their own activity profiles. Thirdly, even in the same environment a user's behavior changes over time to reflect new situations or experiences. As a result, the same person may require different levels of services. Fourthly, in web information retrieval or interface design, the application context is relatively static but in changing pervasive environments the surroundings and events within the environments, e.g. in a train station or shopping mall, and also the network connectivity, e.g. communication bandwidth 
are all dynamic which not only impact on the required services but also the way such services are delivered.

To address these unique characteristics of pervasive context-aware applications as described above, this paper contributes to knowledge in three aspects. Firstly, it introduces a hybrid approach to create and adapt user models based on user behaviours. Secondly, it develops usage data mining methods to learn a user's changing behavior and extract longitudinal patterns. Thirdly, it introduces a service-oriented personalisation framework enabling personalisation services for service personalization.

The remainder of the paper is organised as follows: Section 2 discusses existing related work and highlights the key knowledge contributions. Section 3 introduces the overall conceptual system architecture followed by a detailed description of underlying technologies for the main components, in Section 4. Section 5 outlines a case study including the details of the implementation, testing and evaluation. Section 6 concludes the paper with a brief discussion on future work.

\section{Related work}

Personalisation has been widely studied in web information retrieval [1] and interface design [2]. In user modelling there are two main approaches. One is to apply statistic or probability analysis methods to perform data mining to extract user models from user behavioural data [3]. The other is the knowledge-based user modelling approach which makes use of knowledge engineering techniques, e.g. formal knowledge acquisition, modelling and representation, to build user models. This approach, in particular ontological user modelling, has attracted increasing attention for user modelling of context-aware applications due to their interoperability and ability for knowledge sharing and reuse across several application domains, e.g. COBRA-ONT [4], OntobUM [5] and UPOS [6]. Regarding personalisation mechanisms there are currently three broad categories of techniques, namely case-based personalisation [7], collaborative filtering [6] and rule-based reasoning [8]. Existing approaches to personalisation have three main drawbacks. Firstly, current user models are normally created as a one-off activity leading to fixed user models. It is usually the case that user behaviours change over time, e.g. previously required help is not needed as the users gain knowledge, experience and capabilities. In this case previous user models will not reflect the exact needs of users. Secondly, current user modelling and personalisation usually target one application scenario with relatively stable application contexts, which did not take into consideration the changing service needs and application context. Thirdly, current personalisation functions have been mainly implemented implicitly in a stand-alone manner. It is difficult for them to be shared and reused by distributed pervasive applications which adopt more and more smartphone frontends. To date, little work has been undertaken to fully address these challenges which have originated from the unique characteristics of service personalisation of pervasive context-aware applications for mobile users.

Consequently, the novelties of the proposed approach are three fold. Firstly, user modelling is not a one-off endeavor, however, more an iterative process which will start by developing ontological user models through knowledge engineering techniques and then enhance the models incrementally and dynamically through behaviour learning. Secondly, personalisation will take into consideration the application context and changing environments, e.g. network connectivity, which are unique to pervasive computing in mobile environments. In addition, the proposed approach will be implemented in a service-oriented architecture, i.e. all user models and personalisation mechanisms will be wrapped as personalisation services, thus making it applicable to wider pervasive context-aware applications.

\section{The system architecture}

Figure 1 presents the system architecture of personalisation services for service personalisation within context aware pervasive systems. The architecture aims to enable and support personalisation features of multiple pervasive applications for multiple users in a diversity of pervasive environments, using smartphone frontends. As can be seen in the client side of the architecture there exist many pervasive applications in intelligent pervasive environments such as smart homes, smart hospitals, conferences in addition to open-world use scenarios such as travelling and shopping. For each application there are many users and each may have different needs and desires. To address the diversity of applications and the heterogeneity of users, traditional approaches to personalisation will be not effective. Rather than providing a user model and personalisation mechanism for each application and for each user, it will be more scalable and realistic to provide personalisation features as services. In this case, context information of an application and the interaction usage data between a user and corresponding applications will be monitored through various sensors from the pervasive environment and the application frontend interfaces. The collected behaviour and usage data will be stored locally and transferred to remote servers for advanced processing. Based on the results of data analysis, users' behavior and usage patterns can be extracted which can in turn be used to update user models or directly inform 
service adaptation either in terms of the content or interfaces.

The services in the server-side of the architecture interact with each other and also the client-side functions to provide enabling technologies for personalisation and adaptation of application services. The User Profile Services contain user models of various users and a repository of instantiated user profiles which are supposed to be used for personalisation of different pervasive applications. Initial user models will be created through ontological engineering as user ontologies, which will be later updated based on a user's behaviour analysis in the Data Analysis Services. The Usage Data Services consists of the usage data models of an application and its recorded service usage data, including the configuration of the application interfaces. Usage data will be collected from the application frontends in the client side, stored locally and transferred to a remote server in an asynchronous manner, e.g. in order to save power or use better communication bandwidth. The Context Services will be composed of contextual information systems and a context analysis module. The former will record and store low level contextual information collected from sensor data in the environments of pervasive applications, and the latter will analyse the low level contextual data to extract high level meaningful context in terms of application characteristics and scenarios. All User Profile Services, Usage Data Services and Context Services will support recording, storage, retrieval, query and search functions.

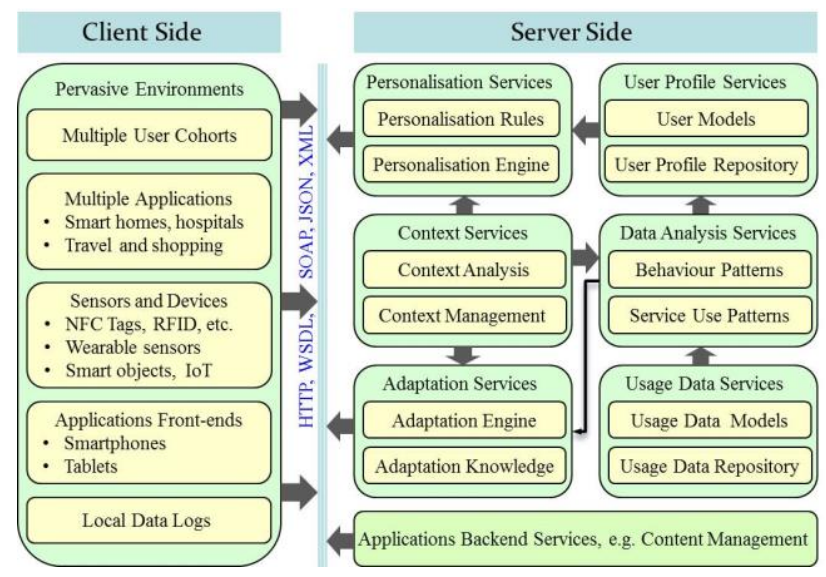

Figure 1. The system architecture of personalisation services

Central to the architecture are the Data Analysis Services, Personalisation Services and Adaptation Services. Data Analysis Services play two roles. One is to analyse the user's behaviour based on high level context, such as daily activities in a smart home environment. Behaviour analysis will be able to extract a user's behaviour pattern and identify the change of a user's behaviour. This will, in turn, be used to update user models in the User Profile Services. The second role is to analyse the usage data of a service, e.g. which functions have been used in which context. The analysis will extract service usage patterns, e.g. the most frequently used feature(s), the volume or font size of the adopted user interfaces. Such findings will then be used to customise the manner in which a service is delivered. The Personalisation Services consists of domain-dependent, application-specific personalisation knowledge and a reasoning engine. The personalisation knowledge, e.g. rules, will be captured and modelled based on application characteristics and scenarios. The engine will take as inputs user models, user application requests and the context, and then perform reasoning over the personalisation rules. It will then inform applications, e.g. backend services such as a content provision system, of the personalised ways of a service being delivered. Similar to Personalisation Services, Adaptation Services contain application specific adaptation rules and an adaptation reasoning engine. Adaptation will mainly address issues relating to applications in changing environments. As such, it will take inputs based on the application context and/or user application requests, and will then perform reasoning over the adaptation rules. It will subsequently adapt the manner in which a service is delivered based on the situation. For example, consider a content service that is configured to provide a video based instruction to a user, based on the user's personal profile, i.e. the user prefers video. Nevertheless, the system detects that the available internet connectivity is poor and as a result the adaptation service adapts the delivery of the content from video to audio or text.

\section{User modelling, personalisation \& behavior learning}

The presented approach and the service-oriented architecture has been considered in the EU funded MobileSage project ${ }^{1}$. MobileSage intended to develop help-on-demand (HoD) services for elderly people, which allow them to carry out and solve everyday tasks and problems in the self-serve society when and where they occur, just-in-time. A typical example scenario is that a user travels within a foreign country and requires assistance to operate a ticket machine to buy a ticket, e.g. in an airport or train station, without understanding the local language. The hypothesis is that the ticket machine is a smart object equipped with Near Field Communication (NFC) or Quick Response (QR) code technologies [9]. When users use the HoD services, deployed in their smartphones (as an app) to interact with the smart ticket machine, the HoD service will retrieve instructions on how to buy a ticket from a content management system in the cloud. The instructions could be

\footnotetext{
1 The MobileSage project http://www.mobilesage.eu/
} 
delivered in different modalities, e.g. video, audio or text, or presented in different interfaces, e.g. layout, volume or font size, based on the user's profile, e.g. preference and capabilities, and the situation of the point of help. The following describes the underlying technical details of the personalisation services and Section 5 outlines the implementation details of the overall system.

\subsection{User profile modelling and personalisation mechanism}

Based on MobileSage application scenarios, three cohorts of end users had been considered in terms of their education background, medical conditions and cognitive capabilities. The user modelling tool PERSONAS was then used to characterise the main concepts and relationships of each cohort which leads to the conceptual user model (refer to Figure 2). To accommodate the diversity of pervasive applications in different environments, the conceptual user models have covered a wide range of entities and relationships. While conceptual models can be serialised in any format, we have used the ontological engineering tool Protégé to develop computational user models, which are subsequently represented in OWL (refer to Figure 3).

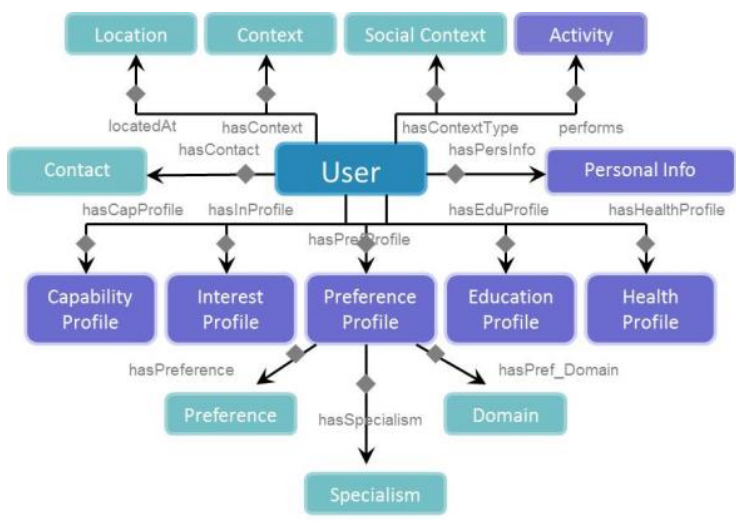

Figure 2. Conceptual user profile models

To provide personalised services it is necessary to specify what services should be provided and how they should be delivered or presented for a specific type of user. Such knowledge is usually domain dependent and application specific. As a result, we have conducted knowledge acquisition in the context of the MobileSage HoD application scenario and extracted personalisation knowledge. Though there are different ways of representing personalization knowledge as discussed earlier, in MobileSage we have adopted Semantic Web Rule Language (SWRL) for personalisation knowledge representation (refer to Figure 4). This is in line with ontological user model representation, thus facilitating assimilation and combination of both semantic and rule based reasoning. Both user models and personalisation rules are stored in native OWL files.

Rules only establish cause-effect relationships. A reasoning engine is required to decide if the pre-conditions of a rule are met, thus leading to a consequence. In addition, the reasoning engine also needs to decide if the consequence of a previously fired rule results in another rule being fired. In this study we used an existing open-source semantic reasoning engine called Pellet [9] as the personalisation engine.
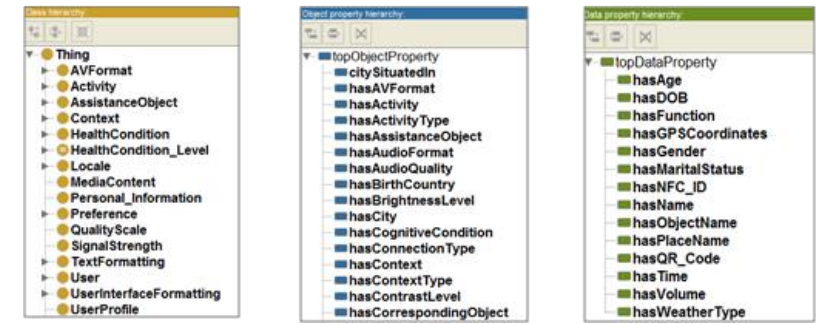

Figure 3. Computational ontological user models

UserProfile(?up), hasHealthCondition(?up, Blind), hasPrimarylanguage(?up, ?lang) $\rightarrow$ HelpDelivery(PlayAudio), UserProfile(?up), hasPrimary Language(?up, None) $\rightarrow$ has hefaultMediaLanguage(?up, English) UserProfile(?up), hasPrimaryLanguage(?up, ?lang) $\rightarrow$ MediaContent(Imagery), hasMediaLanguage(Imagery, ?lang) UserProfile(?up), hasPreferredMediaContent Type(?up, Text) $\rightarrow>$ HelpDelivery(Display Text), hasMedia Type(Display Text, Text) UserProfile(?up), hasHealthCondition(?up, Deaf), hasPreferred TextColour(?up, ?c) $\rightarrow$ HelpDelivery(Display Text),
hasMedia Type(DisplayText, Text), has TextColour(Display Text, ?c), hasTextSize(Display Text, TextSize_18)

Figure 4. SWRL based personalisation rules for $\mathrm{HoD}$

\subsection{Usage data modelling and adaptation mechanism}

Usage data are closely related to the service's functions and features of the applications concerned. In MobileSage we have characterised HoD services into four levels of interactions. Level One refers to all general user interactions such as device events and user interface (UI) events. Device events encompass any user interaction with the hardware of the Smartphone, such as Volume, Back and Menu button presses whilst UI Events are events in which the user has interacted with the UI of the HoD. Level Two refers to service interactions, e.g. what services the user has accessed and also what information or user input have been provided. For example, if the user enters the Search service and searches for restaurants, the Usage Log would record this as a serviceEvent, including what service was accessed, in this instance Search, and what the user's input was, i.e. "restaurants". Level Three refers to user profile interactions, i.e. which user profile properties have been accessed or changed, and the new values. For example, the user modifies the screen brightness, this would be logged as a profileEvent with the identifier "System_Profile_Brightness" and the new value, i.e. brightness level. The final level of event capture involves communication with the Content Management System (CMS). Level Four collects data in the context of "Server Response". When a service is accessed and a request 
for content is made to the CMS the request and a summary of the CMS response is recorded. These events summarise the service requesting content in addition to the user input. While the exact contents of the response can be recorded, a summary of the response is recorded too, which also specifies what modality is returned.

For each level of interaction a data model (refer to Figure 5) is developed to capture the usage data at the level. All usage data is stored locally on the device itself in an SQLite Database, which is later synchronised into a remote server (as described in Section 5.1). We have developed various data analysis methods to mine the usage data to extract user behaviour patterns. These include the general service usage, e.g. the most used functions, the preferred interface configuration and location aware user interfaces (UI). Figure 6 displays the k-means clustering algorithm to determine the centroid of the locations that specific services were used based on the GPS information.

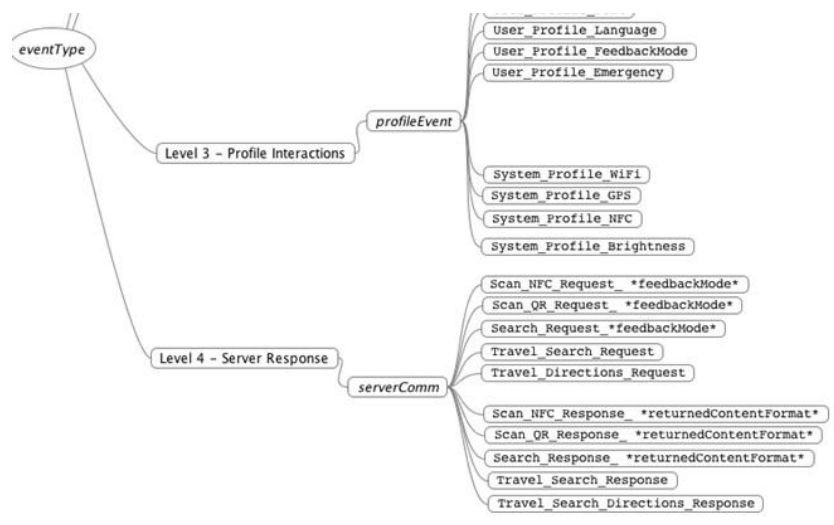

Figure 5. An excerpt of event types and their correspondent models

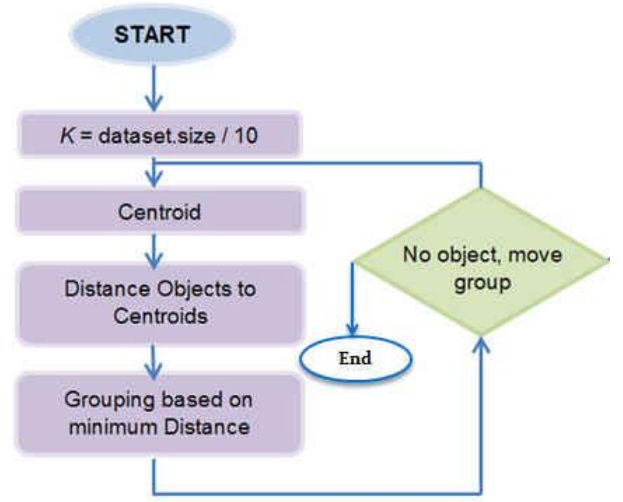

Figure 6. The kMeans algorithm for centroid extraction

\section{Implementation and evaluation}

We have partially implemented the personalisation services in the context of MobileSage project, as presented in Figure 7. The client side is the HoD services, which are implemented as a smartphone app deployed in an Android smartphone. HoD offers a number of services that are made available through an intuitive, adaptive UI [9]. The server side includes four personalisation related services and also a Content Management System which provides content to the HoD application. All MobileSage assistive material is persisted at the CMS and can be provided in a range of different modalities including text, audio and video.

The four personalisation related services have been implemented in a REST-based (REpresentational State Transfer) software orientated architecture (SOA). The underlying technologies for User Profile Services and Personalisation Services include user ontologies, a set of pre-defined SWRL rules, and the employment of the Pellet reasoner. Semantic content such as instantiated user models and rules are managed through OWL-API ${ }^{2}$. Usage data models and collected usage data are managed through SQLite on each client-side device while MySQL is used centrally on the server-side. Java web service development tools, specifically the JAX-RS - the Java API for RESTful web services and Jersey - an open-source, production quality framework, have been used to develop the four services. The development and testing environment included Eclipse Juno, MySQL Server 5.1 and Apache Tomcat 7.0.34. Messaging between services and client-side HoD have been represented as Java classes which are then mapped to JSON format by using the Google Gson library. The Android Developer Tools IDE supported development of the HoD Android application.

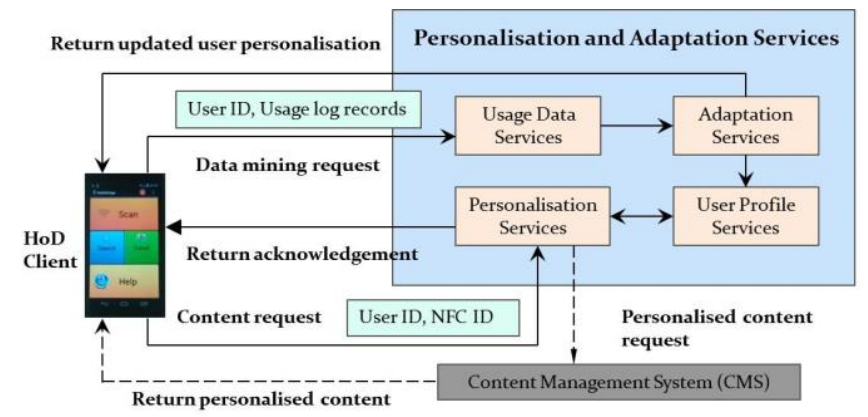

Figure 7. Implementation of the personalisation services

\subsection{Testing and evaluation}

The presented approach and developed underpinning technologies have been tested and evaluated in the Smart Environment Research lab at the University of Ulster in terms of assessing the efficiency and correctness of the HoD

\footnotetext{
2 Open source OWL API http://owlapi.sourceforge.net/
} 
services and personalisation services. Three participants with different user profiles, as shown in Table 1, were instructed to use the HoD services to (1) seek help on purchasing a train ticket from an automated ticket machine and to (2) enable personalised route directions from a specified location to another place of interest. User 1 and 2 tested the first scenario while user 3 tested the second scenario. For the second scenario, over a period of twenty-four hours, a number of service activations were initiated through the HoD including search, scan, help and travel query terms. This activity was modelled to reflect a typical usage scenario of the HoD over a daily period.

\section{TABLE 1. THE THREE PARTICIPANTS' USER PROFILES}

\begin{tabular}{|l|l|l|l|l|l|l|l|}
\hline ID & Profile Name & Media Type Preference & \multicolumn{1}{|c|}{ Health Status } & Sex & Default location & Age & Language Preference \\
\hline $\mathbf{1}$ & Jane & Audio & Vision Impairment & Female & Northern Ireland & 55 & English \\
\hline $\mathbf{2}$ & Jack & Text & Hearing Impairment & Male & UK & 37 & Spanish \\
\hline $\mathbf{3}$ & Elizabeth & Video & None & Female & Northern Ireland & 24 & English \\
\hline
\end{tabular}

To test personalisation and adaptation the HoD usage data were collected and subsequently sent to data analysis services for pattern recognition, which in turn provided updated user models. Table 2 displays an example of the JSON representation of an updated user personalisation response.

To test performance, we recorded the formats of retrieved specific media and the time taken to retrieve information from the HoD service, as shown in Table 3. Initial results have shown that all returned media types match the ground truth of media preference of user profiles, and also the impact of the WiFi or 3G/GSM speed on service performance.

\section{TABLE 2. EXAMPLE USER PERSONALISATION RESPONSE}

\section{\{"searchClusters": ["54.80232415814548,-5.683293549103988"]}

"travelClusters":["54.827482000000025,-5.648274200000002","54.827482000 "nfcClusters":["54.827482000000025,-5.648274200000002"],"qrClusters":[],

"addToFavourites":\{"search":["Versailles"],"travel":["Giverny"]\},

"serviceActivationsLast $24 ":\{$ "search": 9, "scan": 2 ,"help": 0 "travel"::0\},

"serviceActivations":\{"search":17,"scan":22,"help":16,"travel":12\}\}

TABLE 3. TESTING AND EVALUATION EXPERIMENT RESULTS

\begin{tabular}{|l|l|l|l|l|l|l|}
\hline ID & Media Preference & Returned Media Type & WiFi Speed ${ }^{1}(\mathrm{kbps})$ & GSM Speed (kbps) & Time WiFi (ms) & Time GSM (ms) \\
\hline $\mathbf{1}$ & Audio & Audio (4.7MB mp3) & DL 15267; UP 3861 & DL 2488; UP 1433 & 6066 & 8384 \\
\hline $\mathbf{2}$ & Text & Text & DL 5035; UP 6563 & DL 780; UP 1524 & 2245 & 3870 \\
\hline $\mathbf{3}$ & Video & Video (8.7MB 3gp) & DL 13737; UP 16171 & DL 2319; UP 1512 & 6512 & 9532 \\
\hline
\end{tabular}

\section{Conclusions}

This paper proposed an integrated approach and its associated service oriented system architecture for service personalisation of pervasive systems in smart environments. Relevant underpinning technologies such as behaviour analysis and personalisation have been developed based on semantic modelling, representation and reasoning. These services have been prototyped using the latest semantic technologies and service oriented software engineering, and further evaluated in well-designed use scenarios. Initial results have shown that the technologies and service oriented system are working. While further evidence will await large-scale evaluation in real world context with real users, current findings have proved the approach to be viable.

\section{Acknowledgements}

This work was undertaken as part of the EU AAL funded MobileSage project (ref: AAL-2011-3-50). The authors gratefully acknowledge the contributions from the members of the MobileSage consortium.

\section{References}

[1] Anand S. and Mobasher B., Intelligent Techniques for Web Personalization, Lecture Notes in Computer Science, Vol.3169, pp 1-36, 2005.

[2] Weld D.S., Anderson C., Domingos P., Etzioni O., Gajos K., Lau T., Wolfman S., Automatically personalizing user interfaces, Proceedings of the 18th IJCAI conference, p.1613-1619, 2003.

[3] Gallacher S., Papadopoulou E., Taylor N., Williams M.H., Learning user preferences for adaptive pervasive environments: An incremental and temporal approach, ACM TAAS 8(1): 5, 2013.

[4] Chen H., Finin T., Joshi A., An Ontology for Context Aware Pervasive Computing Environments, The Knowledge Engineering Review, 18, 197-207, 2003.

[5] Razmerita L., Angehrn A., Maedche A., Ontology Based User Modeling for Knowledge Management Systems, User Modeling, 148-148, 2003.

[6] Sutterer M., Droegehorn O., David K., UPOS: User Profile Ontology with Situation-Dependent Preferences Support, In Advances in Computer-Human Interaction, pp. 230-235, 2008.

[7] Bettini C., Brdiczka O., Henricksen K., Indulska J., Nicklas D., Ranganathan A., Riboni D., A Survey of Context Modelling and Reasoning Techniques, Pervasive and Mobile Computing, 6, 161-180, 2010

[8] Viviani M., Bennani N., Egyed-Zsigmond E., A Survey on User Modeling in Multi-Application Environments, In Advances in Human-Oriented and Personalized Mechanisms, Technologies and Services (CENTRIC), pp. 111-116, 2010.

[9] Halbach T., and Schulz T., MobileSage - A Prototype Based Case Study Delivering Context-Aware, Personalized, On-Demand Help Content, in Advances in Human oriented and Personalized Mechanisms, Technologies, and Services, pp.1-6, 2013.

[10] Sirin E., Parsia B., Grau B.C., Kalyanpur A., Katz Y., Pellet: A Practical Owl-Dl Reasoner. Web Semantics: science, services and agents on the World Wide Web, vol.5, pp.51-53, 2007. 